\title{
Discernimento del Superiore religioso: la postulazione
}

\section{The discernment of the religious Superior: the postulation}

\begin{abstract}
The main purpose of the present submission relates to the matter of the postulation regulated by the legal disposition expressed in canons 180 e $181 \mathrm{CIC} / 83$. The main emphasis is not placed on the correct application of the canon law, in the case concerning the dispensation granted to who is ineligible to the canonical office or other assignment.

Instead, the Author tries to highlight the meaning and importance that must be given to the discernment undertaken by the collegial Authority in the case of election. The article presents the certain conditions required from the candidate and, at the same time, supplies these criteria, which usually are considered by the Congregation of Institutes of Consecrated Life and Societies of Apostolic Life in the matter of admission of the postulation.
\end{abstract}

\section{Keywords}

Collegiality; religious Superior; canonical office; postulation; impediment; dispensation.

\section{Premessa e circoscrizione tematica}

L'interesse alla problematica di cui alle presenti riflessioni deriva dalla consapevolezza dei continui cambiamenti culturali e sociali, capaci di modificare significativamente il vissuto presente di non poche persone consacrate.

Rileva, in tal senso, lo stato numerico dei consacrati che, conseguentemente, rispecchia l'adeguata preparazione e/o idoneità a ricoprire gli uffici/incarichi richiedenti una peculiare conoscenza, competenza, professionalità ed esperienza. 
Considerato che gli Istituti di Vita Consacrata e le Società di Vita Apostolica ${ }^{1}$ del vecchio mondo vivono, non di rado, una progressiva quanto drammatica diminuzione numerica dei propri sodali, la previsione di un ufficio e/o un incarico richiede dal Superiore competente di abbracciare in un'ampia panoramica la globalità del vissuto dei consacrati sia in una circoscrizione dell'Istituto sia nella sua totalità. Tale compito serve per individuare le eventuali circostanze qualificabili come impedienti la designazione di un sodale ad esercitare la potestà annessa allo stesso ufficio e/o incarico ${ }^{2}$.

In quest'ottica, la decisione di conferire l'incarico non deve essere mai meccanica quanto, piuttosto, il Superiore deve pervenirvi mediante il discernimento intenzionale, nel rispetto dei criteri della postulazione stabiliti dal diritto universale e dall'Autorità competente cui spetta l'ammissione della petito Oratori. Viene in evidenza, a tal proposito, la necessità di individuare dei requisiti minimi - obbligatoriamente riscontrabili nella persona postulata - e di prefissarne il contenuto specifico in modo che il Superiore collegiale o meno, valutando quanto emerso dall'attività discernimentale, proceda ad ulteriora o scelga un'altra modalità decisionale.

Ciò posto, maggior attenzione dottrinale verrà riposta non tanto nell'esame del corretto espletamento procedurale - previsto ai cann. 181 e 182 CIC/83 -, quanto, piuttosto, nella fase precedente attraverso la quale il Superiore religioso avverata la necessità di postulare - provvederà a misurarsi con la realtà dei fatti in modo da intuire gli sviluppi delle proprie decisioni e prospettare le eventuali soluzioni nell'ipotesi della negata ammissione del candidato. Interessa dunque la problematica connessa alla postulazione in chiave dell'attività discernimentale.

L'obiettivo da perseguire dovrebbe, sempre, tener presente la ricerca del bene sia dell'Istituto sia della Chiesa e solo qualora il bene particolare dello stesso Istituto non contrasti con quello pubblico.

1 D'ora innanzi IVC e SVA.

2 Basti pensare alla provvisione dell'Ufficio di un Superiore maggiore o il Supremo moderatore, specie presso gli Istituti femminili claustrali in cui, il mancato scambio generazionale, potrebbe gravamente determinare la scelta di tale figura, rimanendo, in effetti, costretti a chiedere detto intervento alla Santa Sede. Lo stesso si dica quanto agli Istituti maschili considerando l'ipotesi di designazione un membro ad un Ufficio/incarico richiedente una determinata qualifica professionale. 


\section{Superiore religioso: la collegialità}

Premesso che la postulazione è una variante dell'elezione ${ }^{3}$, il dettato legislativo vigente in materia implica l'esistenza della partecipazione di tre soggetti giuridici ognuno dei quali deve possedere le qualità richieste dal Legislatore.

Attese le premesse, interessa maggiormente la figura giuridica del Superiore che forma insieme agli altri - aventi il diritto di voto e ne abili ${ }^{4}$ - un collegio la cui esistenza ed azione costituisce una condicio sine qua non dell'intero istituto giuridico. Il Legislatore omette l'ipotesi in cui la designazione del candidato ad un ufficio/incarico spetti al Superiore che, individualmente e personalmente, esercita la propria potestà di governo nel senso che lo stesso sia privo di qualunque supporto proveniente da una consultazione/un consiglio.

Nell'ipotesi della postulazione, sebbene non si tratti di un'azione collegiale sic et simpliciter ${ }^{5}$, tuttavia, occorre l'esistenza di un corpo elettorale/votante presieduto da un presidente il quale assicura, perlomeno, la discussione riguardo all'idoneità e/o capacità del sodale da designare.

Il Superiore religioso, quanto agli affari di maiores momenti dell'Istituto, nella stragrande maggioranza di casi, rimane vincolato a quanto espresso dai votanti, membri del proprio Consiglio. Limitatamente al consenso occorre rilevare che, spetta, unicamente, al Superiore procedere ad ulteriora tenendo presente tutti gli elementi rilevanti per il caso ${ }^{6}$.

Detta attività è tratto specifico dei Capitoli provinciali, generali e quelli dei monasteri sui iuris, cui scopo primario è eleggere i titolari degli uffici capitali dell'Istituto o della Sua parte, provvedendo, al contempo, al conferimento degli altri incarichi e/o ad un'efficiente soluzione delle questioni discusse in sede del medesimo Capitolo?.

3 Cfr. J. Miñambres, Postulación del candidato, in: J. Otaduy - A. Viana - J. Sedano (ed.), Diccionario General de Derecho Canónico, vol. VI, Aranzadi, Navarra 2012, p. 280; A. Viana, Organización del gobierno en la Iglesia, Eunsa, Pamplona 2010, p. 102-103.

4 Cfr. can. 171, \$1 CIC/83.

5 In tal senso, la fattispecie risulta inesitente nel Codice del 1983. Detta soluzione sarebbe possibile qualora fosse prevista solo dalle Costituzioni dell'Istituto.

6 Cfr. can. 127, $\S 1$ e 2 CIC/83.

7 Cfr. D. Andrés, I Capitoli religiosi. Autonomia, potestà e rappresentanza con particolrae riguardo al diritto di petizione, in: P. Gherri (ed.), Responsabilità ecclesiale, corresponsabilità e rappresentanza. Atti della Giornata Canonistica Interdisciplinare, Lateran University Press, Roma 2010, p. 178. 
In quest'ottica, preme rilevare che il diritto universale assegna la possibilità di governare ai Superiori collegiali ma in veste straordinaria ${ }^{8}$ ragion per cui gli stessi possono formare un vero e proprio corpo collegiale dotato di una potestà suprema responsabile sia per l'autogoverno sia per l'autodeterminazione.

La collegialità è, dunque, una specifica caratteristica dello stile di un'autorevole guida dell'intero Istituto di vita consacrata. Tuttavia, questo non è solo dato dall'esistenza o obbligatorietà dell'istituto del Capitolo nella vita consacrata, ma emerge con evidenza dalla gestione quotidiana del vissuto ecclesiale qualora sia necessario richiamare gli Organi/Organismi ${ }^{9}$ diversi quali veri strumenti di partecipazione nel processo decisionale dell'Autorità.

L'argomento in esame richiama, dunque, il coinvolgimento di tali enti che, realizzando il proprio compito, pervengono ad un quadro, sufficientemente, completo e consapevole in vista della(e) decisione(i) vincolante(i). Lo stesso collegio votante partecipa, attivamente, alla vita di una concreta comunità dei consacrati (giurisdizionale o sui iuris che sia), indipendentemente dal nomen iuris ad esso qualificato.

Inoltre, rileva la dinamica del percorso riguardante la formazione della decisione concreta poiché la stessa richiede, qualora possibile, la massima conoscenza del caso da decidere insieme ad un corretto utilizzo della metodologia lineare di lavoro che consenta l'elaborazione dei criteri di buon governo, ai sensi del can. $50 \mathrm{CIC} / 83$.

\section{Candidato ad un ufficio/incarico}

Richiamando quanto prefissato nella premessa, ora interessa analizzare le prescrizioni del Legislatore riguardo alla persona del candidato.

8 Cfr. can. 631, §1 CIC/83; F. D’Ostilio, L'esercizio della sacra potestà negli Istituti religiosi, Libreria Editrice Vaticana, Città del Vaticano 2000, p. 75. Sebbene il Legislatore nulla stabilisca in ordine alla designazione di un Superiore del monastero sui iuris, tutttavia, l'ordine sistematico del can. $625 \mathrm{CIC} / 83$ permetta di desumere che lo stesso debba essere, piuttosto, eletto non, invece, nominato. Cfr. T. Rincón, Sub cann. 625-626, in: J. I. Arrieta (ed.), Codice di Diritto Canonico e le Leggi complementari, Coletti, Roma 2004, p. 460.

9 Tale distinzione è convinzione personale di chi scrive poichè richiama la doppia possibilità di partecipare al processo decisionale dei Superiori: agli Organi spetta dare il consenso, agli Organisimi, invece, solo il parere. Attese le disposizioni legali di cui al can. 127, §§ 1-2 CIC/83, solo gli Organi partecipano, deliberativamente, nel processo decisionale ed, a volte, dal loro consesno dipende la reale esistenza della decisione ed il suo esito. Cfr. can. 633, §1 CIC/83. 
Affinché si possa parlare della postulazione ed attuarla, il dettato legislativo vigente richiede l'esistenza simultanea di tre condizioni: a mente del can. 180, $\$ 1 \mathrm{CIC} / 83$ il candidato deve essere considerato «più adatto» o preferito ${ }^{10}$ dagli elettori. Inoltre, preme affermare che questi requisiti devono convincere il corpo elettorale in maniera tale da ritenere un sodale - tuttavia impedito ad esercitare la propria potestà - unico soggetto capace e/o preposto ad un ufficio/incarico concreto. Per questo motivo detta ipotesi diventa causa giusta per chiedere la dispensa e, conseguentemente, causa efficiente della postulazione stessa. Inoltre, è ipotizzabile che gli stessi requisiti, in un certo senso, costringano a procedere alla postulazione poiché la stessa sarebbe un'unica ragionevole soluzione emergente dall'effettivo vissuto ecclesiale.

Convocati gli elettori ${ }^{11}$, come dimostra l'esperienza, deve essere noto ai votanti - nel maggior numero di casi - un impedimento imputabile alla persona del candidato ${ }^{12}$. Lo stesso non è solo nominato come potenziale titolare dell'ufficio/incarico, di solito in maniera informale, ma, soprattutto, tra gli elettori si forma una ferma volontà e convinzione che questo sodale sarà votato in vista della provvisione. Senz'ombra di dubbio, non ha alcun senso - come, contrariamente, avviene nell'ipotesi dell'elezione - che l'Autorità verifichi, previamente, l'esistenza dei requisiti necessari in vista della provvisione. Ciò che rileva, piuttosto, è il giudizio prognostico quale espressione rilevante del discernimento insieme alla convinzione basata sui dati concreti che al sodale designato sarà concessa la dispensa. Tuttavia, si deve, sempre, tener presente che la legge canonica non debba essere svuotata da un'eccessiva facilità, se non meccanicità, nel permettere eccezioni ad essa ${ }^{13}$.

Tale atto, imprescindibile per procedere ad ulteriora, può essere raggiunto qualora gli stessi votanti raggiungano il quorum di due terzi di voti, ciò a mente del can. 181, §1 CIC/83. Segue, dunque, che il livello di determinazione tra gli elettori deve essere, davvero, significativo.

${ }^{10}$ È opinione di chi scrive che detta "preferenza" non si riferisce ad un legame familiare di nessun tipo, sebbene la mens Legilatoris voglia definire tali qualità specifiche nella persona del candidato che, nonostante un elemento legale mancante assieme ad una ponderata prognosi positiva, soddisfi in maniera miglior possibile l'effettiva esigenza quanto ad un'autorevole giuda o un diligente espletamento dell'incarico affidato.

${ }^{11}$ Cfr. cann. 166-173 CIC/83.

${ }^{12}$ Cfr. J. Miñambres, Sub can. 180, in: A. Marzoa - J. Miras - R. Rodríguez-Ocaña (ed.), Comentario exegético al Código de Derecho Canónico, vol. I, Eunsa, Pamplona 1997, p. 1023.

${ }^{13}$ Cfr. P. Pavanello, La concessione di grazie: aspettative ed attese, in: J. I. Arrieta (ed.), Discrezionalità e discernimento nel governo della Chiesa, Marcianum Press, Venezia 2008, p. 184. 
Quanto, ancora, all'impedimento definibile come circostanza inabilitante la persona a ricevere gli uffici/incarichi ecclesiali ${ }^{14}$, lo stesso può provenire dalle disposizioni dal diritto universale, particolare e quello proprio ${ }^{15}$. Il testo legale determina dei criteri soggettivi ed oggettivi ${ }^{16}$ la cui esistenza potrebbe assicurare un possibile - quanto potenziale - buon governo di chi sarà chiamato a garantirlo o un diligente espletamento delle mansioni derivanti da un incarico, legittimamente, conferito.

\section{Criteri d'ammissibilità della postulazione}

Chiarite le questioni inerenti la figura del candidato, occorre ora spendere qualche parola sugli altri criteri che, previa ricorrenza, costituiranno un fondamento dell'ammissione di quanto postulato. In tal attività, non si tratta di convincere l'Autorità cui spetta concedere la dispensa, piuttosto, sarebbe opportuno dimostrare alla stessa Autorità un'oggettiva utilità della concessione della grazia frutto di un'argomentazione razionale che la renderà un atto

${ }^{14}$ Come osserva la dottrina, non rilevano gli impedimenti di carattere civile, piuttosto soltanto quelli canonici - provenienti dal diritto divino, naturale e positivo - dai quali l'Autorità competente, solitamente, concede l'atto della dispensa. Cfr. J. García Martín, Le norme generali del Codex Iuris Canonici, Ediurcla, Roma 2006, p. 612-613.

${ }^{15}$ Non va omessa l'ipotesi di un impedimento stabilito dagli Statuti degli Enti canonici quali Associazioni dei fedeli collegate all'Istituto di vita consacrata e dallo stesso promosse, ciò a mente del can. $312 \mathrm{CIC} / 83$. È ipotizzabile che un presidente di tali strutture debba essere un sodale dell'Istituto che promuove un'Associazione ma, anche, debba possedere delle qualità e/o qualifiche particolari.

${ }^{16}$ Cfr. rispettivamente cann. 149-150 e 151-154 CIC/83. In primo luogo, dal candidato si richiede che rimanga nella comunione con la Chiesa (cfr. cann. 96; 204-205 CIC/83) a servizio ed in nome della quale dovrà agire per il bene comune. Questo porta ad escludere quei sodali nei confronti dei quali è stata, legittimamente, dichiarata qualche censura (per esempio cfr. can. 1364, §1 CIC/83; tuttavia, l'eventuale dispensa non debba essere in palese contradizione con la disposizione della sanzione inflitta). Inoltre, lo stesso deve possedere di quanto specificamente richiesto dal diritto per l'ufficio in oggetto. Potrebbe trattarsi dell'età, di un grado accademico, dei legami di parentela, di uno status canonico concreto. L'ipotesi degli IVC e le SVA potrebbe riguardare, anche, gli altri requisiti quali ad esempio un congruo spazio di tempo dopo la professione perpetua o definitiva (cfr. can. $623 \mathrm{CIC} / 83$ ) quale criterio della valida designazione all'ufficio capitale, come anche l'ordinazione presbiterale per quanto riguarda la provvisione all'ufficio del Superiore nell'ipotesi degli Istituti clericali, o altro simile stabilito dal diritto proprio. Non possono essere omessi i criteri oggettivi come la compatibilità d'esercizio (fisico e morale) degli uffici conferiti alla stessa persona (cfr. can. 636, $\S 1 \mathrm{CIC} / 83$ ); l'ipotesi in cui il candidato è privo di voce passiva per qualsiasi incarico pubblico (a motivo di cause particolarmente ed eccezionalmente gravi); la necessaria vacanza di diritto dell'ufficio da conferire; l'eventuale ineleggibilità per raggiungimento del numero massimo di mandati previsti dal testo costituzionale; la necessità di dichiarare l'eventuale possesso illegittimo di un ufficio vacante di diritto ma, non, di fatto, ed altro simile. 
imparziale. In conformità alla corretta stesura e/o preparazione formale degli atti amministrativi, occorrerebbe presentare, perlomeno saltem summariae, i motivi per cui sarebbe opportuno che la concessione fosse fatta. Inoltre, non va trascurato che l'Autorità dispensante possa ammettere richieste in forza della discrezionalità che il dettato legislativo garantisce ad ogni Superiore gerarchico chiamato a scegliere, per compito di ammettere la petitio Oratori, tra varie possibilità ugualmente legittime.

\subsection{Livello codiciale}

Per istituire la valida postulazione, il Legislatore richiede che - oltre quanto già riportato dal can. $180 \mathrm{CIC} / 83$ - si debba raggiungere la maggioranza di due terzi dei voti, validamente, emessi dagli elettori i quali devono costituire la maggioranza assoluta in collegio, in conformità al can. 181, §1 CIC/83.

Il secondo criterio di cui al paragrafo successivo della medesima norma, determina l'utilizzo di una formula di postulare tenendo presente due ipotesi tra le quali, in ragione della presente riflessione, interessa mettere in rilievo quella che contiene la doppia manifestazione di volontà degli elettori. In altri termini: il collegio, al di là dell'apparente formalismo di giungere quasi all'unanimità di voti, esprime la volontà di scegliere il sodale e di sollecitare la dispensa dall'impedimento imputabile allo stesso candidato. Solo in questa maniera diventa efficace, giuridicamente parlando, il conferimento di quanto accordato.

Contrariamente alle affermazioni di alcuni Autori ${ }^{17}$, la suddetta modalità, consapevolmente e coscientemente adoperata, fa sì che lo stesso corpo elettorale non rinunci al suo diritto di elezione poiché - come recita il can. 183, §3 CIC/83 - la competenza ad eleggere, indipendentemente dall'esito della richiesta pro gratia, spetta unicamente al collegio. Vi è di più: l'analisi puramente esegetica della norma consente di affermare che l'Autorità - destinataria della postulazione - dispensando dall'impedimento non abbia nessun diritto di avocare a se la competenza di conferire un ufficio o quant'altro richiesto.

\subsection{Prassi della Congregazione IVC e SVA ${ }^{18}$}

Il collegio che intende attuare la postulazione deve tener presente che l'Autorità, decidendo la concessione della dispensa, dovrà interrogarsi non solo circa il

${ }^{17}$ Cfr. V. De Paolis, La vita consacrata nella Chiesa, Marcianum Press, Venezia 2011, p. 359.

${ }_{18}$ Detto Dicastero è competente in ordine alla concessione delle dispense inoltrare dagli IVC e le SVA solo di diritto pontificio. Analogamente, gli IVC e le SVA di diritto diocesano presentalno 
corretto espletamento del dettato legale da parte dell'oratore ma, soprattutto e principalmente, dovrà valutare l'opportunità della grazia da concedere.

Detta valutazione, in primo luogo, si basa sulla comprensione globale della situazione inoltrata al Dicastero interpellato a provvedere in maniera più opportuna possibile. In effetti, l'ipotesi frequente - avverabile nella stragrande maggioranza dei casi - concerne la dispensa circa la possibilità di governare l'Istituto per un ulteriore mandato provocando, tuttavia, conflitto con il diritto proprio. La Congregazione per IVC e SVA, adducendo le motivazioni contrarie all'inoltrata richiesta della postulazione di una religiosa di 86 anni, affermava che nonostante il buon stato fisico e mentale della religiosa, non vi fosse nessuna garanzia che la candidata sarebbe in grado di governare con medesima forza e lucidità per un ulteriore mandato ${ }^{19}$. Non si considerava come criterio decisivo dell'ammissione la stima delle sorelle e della gerarchia ecclesiastica. Inoltre, lo stesso Dicastero rendeva evidente la disposizione del can. 624, $\S \S 1$ e 2 CIC/83 come principio generale al quale dovrebbe adeguarsi ogni Istituto nell'ipotesi dell'elezione del Moderatore supremo e/o Superiore maggiore. Inoltre, un ulteriore motivo di diniego della dispensa proveniva dallo stato numerico dell'Istituto che, al momento della postulazione, contava 470 religiose. Considerando detta cifra, da una parte risultava poco probabile l'effettiva mancanza di un'altra religiosa idonea e capace a governare e, dall'altra emergeva una probabilità che, istituendo la postulazione, si volesse raggiungere obiettivi diversi da quelli del bene pubblico.

La fattispecie appena riportata si collega ad un altro criterio con cui si avalla la Congregazione. Si tratta della postulazione, in vista del conferimento dell'ufficio della Superiora generale, onde la candidata sia la Fondatrice dell'Istituto. Come regola generale, il Dicastero suggerisce che dopo l'approvazione pontificia, l'Istituto dovrebbe affrancarsi dal proprio Fondatore «al fine di evitare l'instaurazione di un'eccessiva e, comunque, inopportuna dipendenza dalla persona che è stata mediatrice del suo carisma $)^{20}$.

Atteso il dettato legislativo di cui al can. 624 CIC/83, il Dicastero ammette nella prassi la possibilità di apporre alla propria decisone graziosa, una clausola, espressa in termini hac vice dumtaxat oppure pro ultima vice ${ }^{21}$ qualora si voglia

la postulazione al Vescovo diocesano.

${ }^{19}$ Cfr. O. Manzo, La postulazione nella prassi della Congregazione per gli Istituti di vita consacrata e le Società di vita apostolica, "Sequela Christi” 2 (2012), p. 182-183.

${ }^{20}$ Congregazione per gli Istituti di Vita Consacrata e le Società di Vita Apostolica, Rescriptum, Prot. N. 11072/1998, in: O. Manzo, La postulazione nella prassi della Congregazione..., p. 184.

${ }^{21}$ Cfr. O. Manzo, La postulazione nella prassi della Congregazione..., p. 185. 
conferire un ufficio alla stessa persona in determinate circostanze. In realtà, si tratta di una condizione secondo la quale lo stesso sodale sia impedito ad homine per un'altra postulazione e, sebbene successivamente fatta, non sarà ammessa per alcun motivo. Lo stesso diniego potrebbe avverarsi nell'ipotesi della postulazione per un altro mandato consecutivo rispetto a quello postulato dagli elettori.

Sulla falsariga di quanto appena affermato, esiste nella prassi della Congregazione, l'ammissione parziale della postulazione. Si tratta dell'ipotesi in cui vi è, davvero, una grave mancanza di sodali idonei ad assumere certi uffici e/o incarichi. Tale circostanza è legata ad una situazione urgente cui occorre provvedere quanto prima. La parzialità proposta dal Dicastero si traduce, in effetti, nell'abbreviare la durata del mandato richiedendo, al contempo, la deroga dalle disposizioni delle Costituzioni. In tal caso, come ben osserva O. Manzo, potrebbero sorgere ulteriori problemi: i Consiglieri del Moderatore supremo (o altri titolari degli uffici ne consessi) ${ }^{22}$, dopo la scadenza del mandato "abbreviato" potrebbero attribuirsi il diritto di portare a termine l'intero mandato per il quale sono stati eletti dal Capitolo.

Simile fattispecie è oggetto di risposta del Dicastero alla postulazione parziale in cui la Superiora generale, scaduto il mandato con tale clausola, viene nuovamente postulata dalla Vicaria generale assieme al Consiglio, in vista della proroga di un mandato stabilito in tal senso. Le motivazioni riportate a favore della proroga non sono state considerate sufficienti, ragion per cui il Dicastero ha ordinato una nuova elezione nell'Istituto in cui la stessa religiosa postulata non poteva essere presa in considerazione per detto ufficio capitale ${ }^{23}$.

Ne consegue che il maggior numero d'ipotesi finora considerate riguardano le situazioni in cui i sodali votanti si riuniscono in un Capitolo elettivo. Tuttavia, è verosimile che la postulazione possa essere presentata da un collegio diverso di Autorità inferiore ad un Organo capitolare. In tal ipotesi, il Dicastero romano, valutando l'opportunità inerente l'ammissione o meno della richiesta, esige la previa opinione del Moderatore supremo con il Suo Consiglio quanto al merito della questione postulata ${ }^{24}$. Si ritiene che il parere del Governo generale dell'Istituto, sebbene privo di carattere deliberativo, si sostanzi in quanto detto

\footnotetext{
${ }^{22}$ Solo nell'ipotesi in cui non fosse stabilita la durata di tale ufficcio e, cioè, qualora non decadessero assieme alla cessazione del Moderatore supremo.

${ }^{23}$ Cfr. Congregazione per gli Istituti di Vita Consacrata e le Società di Vita Apostolica, Rescriptum, Prot. N. 10694/1998, in: O. Manzo, La postulazione nella prassi della Congregazione..., p. 186.

${ }^{24}$ Cfr. O. Manzo, La postulazione nella prassi della Congregazione..., p. 180.
} 
e/o raccolto a livello provinciale o di una concreta casa religiosa, ad esempio di un monastero sui iuris.

A tal proposito, occorrerebbe corredare una tale richiesta adducendo, soprattutto, gli argomenti, le prove e/o i documenti qualora necessario per motivare a sufficienza ed in modo ragionevole l'avvio della postulazione. Pervenuta la postulazione al Governo generale, lo stesso, basandosi sul materiale ricevuto e seguendo la propria prassi e metodologia, potrà considerare quest'atto quale supporto presso la Curia Romana.

\section{Discernimento intenzionale}

Le riflessioni appena riportate mettono in risalto i momenti fondanti e/o decisivi dell'istituzione della postulazione. L'importanza di quanto si vuole raggiungere spinge il collegio elettorale, da una parte ad osservare le disposizioni del Legislatore in oggetto e, dall'altra, ad interrogarsi sull'opportunità operativa di tale modalità procedurale ed i suoi possibili sviluppi, avverata l'ammissione della richiesta. L'Istituto, rappresentato dagli elettori, non può provvedere alle proprie necessità (istituzionali, comunitari, pastorali, formativi che sia) con un certo tecnicismo che non si addice ad un evangelico tenore ed una fedeltà dinamica ${ }^{25}$ di vita consacrata o apostolicamente associata. Si tratta, semmai, di uno stile di governo marcato da una sensibilità maggiore atto a valutare e coordinare meglio le risorse di cui si dispone (particolarmente, quelle umane $)^{26} \mathrm{e}$ ad attuare una metodologia che comprenda la complessità dei possibili - quanto potenziali - eventi, rilevanti per l'avvenire dell'Istituto. Il discernimento quale atto di riflessione - mirante alla ricerca della soluzione più adeguata per soddisfare un'esigenza concreta - non deve essere, mai, un metodo fissato a priori, piuttosto, una capacità tecnica provvista di una forte dose d'intuito che abbisogni di conformarsi alle persone che l'applicheranno, alla concreta comunità che sarà destinataria di quanto sottoposto allo stesso discernimento, ed, infine, alle situazioni diverse in cui si è chiamati ad effettuarla.

${ }^{25}$ Cfr. Ioannes Paulus PP. II, Adhortatio Apostolica post-Synodalis Vita Consecrata, 25 Martiis 1996, "AAS" 88 (1996), p. 400-440, nº 37.

${ }^{26}$ Cfr. P. Gherri, Corresponsabilità e diritto: il diritto amministrativo, in: P. Gherri (ed.), Responsabilità ecclesiale, corresponsabilità e rappresentanza. Atti della Giornata Canonistica Interdisciplinare, Lateran University Press, Roma 2010, p. 128-129. 
Il discernimento inteso come metodo e criterio del governo ecclesiale è un percorso che porta a confrontarsi insieme, con passaggi logici ed ordinati, sugli elementi rilevanti del caso (oggettivi e soggettivi) insieme ad un'urgenza decisionale inerente la realtà da ordinare. Tale costanza intenzionale analizza la situazione, la valuta alla luce dei criteri in uso comune ed è finalizzata a formulare un giudizio e ad operare delle scelte adeguate. È intenzionale poiché non serve tanto ad acquisire solo un insieme d'informazioni vere o false, piuttosto, è indirizzata, coscientemente e deliberatamente, ad una soluzione che, fra tante potenzialmente avverabili, potrà garantire la volontà della maggioranza dell'Istituto riguardo alla tutela della propria autonomia che, tuttavia, non è assoluta. Chi è chiamato a discernere intenzionalmente, dunque, deve assegnare il fine stesso all'azione da compiere.

Se da una parte, la decisione d'istituire la postulazione richiede una globale comprensione della problematica cui far fronte, dall'altra però, si sollecita la consapevolezza inerente l'esistenza di parametri legali ed altri - derivanti dalla prassi - la cui mancata osservanza renderà l'intero percorso non solo poco fondato ma, anche, inefficiente.

Tuttavia, il siffatto discernimento non potrà, mai, creare l'impressione che l'attuazione dell'istituto giuridico di cui si discorre - fatta salva l'obbedienza alla ragionevolezza della richiesta ed i criteri stabiliti - sia attuato con l'obiettivo di far permanere i Superiori e/o gli altri titolari di incarichi rilevanti oltre il tempo congruo ed in maniera indebita ${ }^{27}$.

\section{Il giudizio prognostico}

Attesa la determinazione dell'oratore di portare a termine la postulazione con esito positivo, lo stesso Istituto richiedente deve, prudentemente, considerare le varie possibilità, potenzialmente, avverabili in seguito alle concrete vicissitudini.

In primo luogo, si tratta della previa soddisfazione dei fondamentali criteri permettenti una corretta impostazione del procedimento da avviare (elementi materiali e formali). In seguito, occorre la presa di coscienza di quanto stabilito dal diritto universale e quanto seguito dalla prassi della Curia Romana per raggiungere l'obiettivo della richiesta e, cioè, ottenere efficacia giuridica

${ }^{27}$ Cfr. can. 624, $\S \S 1$ e 2 CIC/83; D. Andrés, Le forme di vita consacrata. Commentario teologicogiuridico al Codice di diritto canonico, Ediurcla, Roma 2008, p. 181. 
all'elezione - a modo di postulazione - e, poi, renderla operante ${ }^{28}$. Detti criteri potrebbero far comprendere l'eventuale sviluppo della decisione presa, priva, tuttavia, di carattere definitivo.

A tal proposito, non andrà trascurata la suesposta prognosi nell'ipotesi di un negativo esito decisionale da parte dell'Autorità cui spetta l'ammissione della postulazione a mente del can. $182, \S 1 \mathrm{CIC} / 83$. La norma successiva, al primo paragrafo, prevedendo tale situazione, la risolve solo apparentemente. In realtà, il Legislatore disciplina che il diritto di eleggere ritorna alla competenza del medesimo corpo elettorale, autore dell'istituzione della postulazione. Tuttavia, questo non aiuta, in nessun modo, a risolvere il problema. È l'ipotesi in cui il sodale postulato fosse l'unico candidato ritenuto valevole dagli elettori ad essere, nuovamente, titolare di quanto chiesto. Qualora si avverasse l'ipotesi di diniego dell'ammissione ${ }^{29}$, lo stesso corpo elettorale dell'Istituto dovrebbe provvedere all'accurata ricerca di un altro sodale idoneo e non sempre potrà essere un compito facile e/o pienamente realizzabile. Non può essere esclusa l'eventualità in cui sarà il Dicastero romano a richiedere ulteriori informazioni dettagliate dalle quali sarà possibile comprendere, globalmente, le vere problematiche interne ed indicare le strade cui percorrere per superare delle difficoltà.

L'intento di tale operazione sarebbe finalizzato a proporre un altro candidato agli uffici capitali o altro. Nell'ipotesi contraria, l'Istituto - trovandosi in un punto cruciale della propria esistenza - dovrà, verosimilmente, affrontare il problema della fusione, unione o altre serie modifich ${ }^{30}$ (quanto agli Istituti numericamente piccoli) o dell'imposizione da parte del Dicastero di un sodale - titolare di quanto chiesto - poiché lo stesso non può garantire elementi essenziali della propria vita. Tale considerazione potrebbe provenire dal consiglio paterno dato dalla Congregazione IVC e SVA. La soluzione meno radicale, invece, potrebbe configurarsi come una sollecitazione/un invito ad un'adeguata preparazione di alcuni dei sodali ad esercitare gli uffici più importanti dell'Istituto in modo che la postulazione non diventi un mezzo, quasi, ordinario d'elezione qualora occorre effettuarla ${ }^{31}$.

La prognosi - sebbene macchinosa - permette di intuire i possibili sviluppi delle decisioni prese qualora la stessa si fondi sull'atto del discernimento

\footnotetext{
${ }^{28}$ Cfr. can. 183, §2 CIC/83.

${ }^{29}$ Raramente avverabile secondo quanto previsto dal can. 57, §2 CIC/83.

${ }^{30}$ Cfr. can. $582 \mathrm{CIC} / 83$.

${ }^{31}$ Cfr. O. Manzo, La postulazione nella prassi della Congregazione..., p. 185.
} 
condiviso e, cioè, tra gli elettori, membri pleno iure dell'Istituto. Questa condivisione decisionale, infatti, - qualora praticata come modo ordinario, non casuale, di trattare e/o concludere gli affari di vario genere - crea un certo stile di governo ecclesiale che, mediante l'esercizio di chi ne è titolare, non si pone nei confronti dei destinatari come un assetto impositivo, piuttosto, una soluzione/un programma condiviso ed accettabile in base del vincolo della stessa fede e premura verso la verità. In questo senso il discernimento diventa consiglio istituzionale ${ }^{32}$.

\section{Conclusione}

La lettura delle norme riguardanti l'istituto giuridico della postulazione, fatta insieme alla considerazione dei criteri codiciali, dicasteriali ed alla presa in considerazione sotto il profilo discernimentale consente, ora, di trarre alcune riflessioni conclusive non prive di rilievo e, forse, d'interesse.

a) In primo luogo va osservato lo scarso coinvolgimento e la scarsa attenzione dottrinale in tema. Il meccanismo di postulazione, assolutamente sconosciuto alla quasi totalità degli Organismi ecclesiali, è trattato soltanto dal punto di vista della correttezza procedimentale affinché la designazione dei candidati a quanto richiesto assuma il valore giuridico (cann. 181-182 CIC/83).

b) Inoltre, l'istituto giuridico della postulazione rappresenta una specificità, se non un'eccezionalità ovverosia l'ammissione della postulazione determina una peculiare modalità legale di conferire un ufficio e/o incarico ecclesiale mediante l'elezione. Al contempo, la dispensa - elemento imprescindibile per un valido conferimento di quanto richiesto - viene concessa, anche, in modo specifico poiché implica la deroga al dettato legislativo (universale, proprio che sia).

Sembra che il segno d'eccezionalità accompagni detto istituto da lungo poiché, anche, il Legislatore canonico del 1917 considerava straordinaria la postulazione nel diritto dei religiosi quanto alle elezioni effettuate in sede dei Capitoli ${ }^{33}$.

c) Ammessa detta eccezionalità, è opinione di chi scrive che tale debba rimanere, sebbene il vissuto ecclesiale, specie quello degli IVC e delle SVA

${ }^{32}$ Cfr. P. Gherri, Note sul discernimemto ecclesiale, Lucera (FG) 9 marzo 2012, in: URL: <http:// gherripaolo.eu/lucera_discernimento.pdf> (06.06.2014).

33 Cfr. can. 507, §3 CIC/1917. 
dimostri, spesso ed esattamente, il contrario e, cioè, che gli stessi Istituti ricorrono a detto meccanismo con maggior frequenza, adducendo le proprie motivazioni. Dai documenti pubblicati nel periodico ufficiale della CIVC SVA Sequela Christi, si evince che la postulazione viene impiegata più volte dalle religiose che dai religiosi a dimostrazione di una serie di problematiche di vario genere sottostante a tale realtà (difficoltà formative, psicologiche, mentali, istituzionali etc).

d) L'obiettivo centrale della presente riflessione dottrinale era di suscitare la necessità di esercitare il proprio munus/officium/ministerium di Autorità ponendosi nella condizione di poter discernere in modo tale da poter intuire gli sviluppi delle proprie decisioni alla base di quanto previsto dal diritto universale e stabilito come una razionale prassi dicasteriale. Quanto a quest'ultima, rileva asserire che il Dicastero competente nell'ipotesi contemplata non dispone di alcun tipo di particolari criteri oltre quelli che detta la ragionevolezza insieme al senso comune del buon governo ecclesiale.

e) Nonostante l'indubbia impressione che si tratti soltanto di una proposta di livello ancora provvisorio è, comunque, rilevabile, forse, la novità dell'approccio discernimentale al tema della postulazione.

\section{Bibliography}

Andrés D., I Capitoli religiosi. Autonomia, potestà e rappresentanza con particolrae riguardo al diritto di petizione, in: P. Gherri (ed.), Responsabilità ecclesiale, corresponsabilità e rappresentanza. Atti della Giornata Canonistica Interdisciplinare, Lateran University Press, Roma 2010, p. 153-182.

De Paolis V., La vita consacrata nella Chiesa, Marcianum Press, Venezia 2011, p. 359.

García Martín J., Le norme generali del Codex Iuris Canonici, Ediurcla, Roma 2006, p. 612-613.

Gherri P., Corresponsabilità e diritto: il diritto amministrativo, in: P. Gherri (ed.), Responsabilità ecclesiale, corresponsabilità e rappresentanza. Atti della Giornata Canonistica Interdisciplinare, Lateran University Press, Roma 2010, p. 115-152.

Gherri P., Note sul discernimemto ecclesiale, Lucera (FG) 9 marzo 2012, in: URL: <http:// gherripaolo.eu/lucera_discernimento.pdf $>$ (06.06.2014).

Manzo O., La postulazione nella prassi della Congregazione per gli Istituti di vita consacrata e le Società di vita apostolica, "Sequela Christi” 2 (2012), p. 173-188.

Miñambres J., Postulación del candidato, in: J. Otaduy - A. Viana - J. Sedano (ed.), Diccionario General de Derecho Canónico, vol. VI, Aranzadi, Navarra 2012, p. 280-282.

Pavanello P., La concessione di grazie: aspettative ed attese, in: J. I Arrieta (ed.), Discrezionalità e discernimento nel governo della Chiesa, Marcianum Press, Venezia 2008, p. 179-186. 